\title{
Understanding nursing assistants' experiences of caring for older people in
} pain: The Australian experience.

\author{
Authors: \\ Kristi Holloway, RN, BSc (Hons), Curtin University of Technology \\ Ruth McConigley, RN, PhD, Curtin University of Technology
}

Correspondence:

Kristi Holloway

Western Australian Centre for Cancer and Palliative Care

PO Box U1987

Perth, Western Australia, 6845.

Email: kholloway@bordernet.com.au

Telephone: +61892661760

Fax: +618 92661770 


\begin{abstract}
Objective: This study examined the experiences of nursing assistants (NAs) who have worked with older people in residential aged care facilities (RACFs) who are in pain, to ascertain the role that NAs play in pain management process.
\end{abstract}

Design: A descriptive, exploratory qualitative approach was used to examine NAs' experiences.

Setting: Six NAs employed in three RACFs in regional Western Australia participated in this study.

Methods: In-depth interviews focusing on the NAs' experiences of caring for older people in pain were conducted.

Findings: This study provides an understanding of the care needs of older residents in pain and illuminates the role of NAs in the pain management process. The central role that NAs play in RACFs is highlighted, as are the responsibilities associated with pain management.

Conclusions: NAs may be responsible for initiating and implementing pain management treatments for older residents. This is outside the scope of practice for NAs, therefore it is imperative that the preparation of NAs give them adequate knowledge to perform this role. As well, it may be timely to consider regulation of this large group of workers who are performing clinical tasks in RACFs.

\title{
Key Words
}

1. nursing assistants

2. older people

3. pain

4. residential aged care facilities 


\section{Introduction}

Pain is a common problem for residents in aged care facilities (RACFs) (The Australian Pain Society [APS], 2005). Although pain is not part of the normal ageing process, estimates suggest that between 45 percent (Ferrell, 1995) and 80 percent (Allcock, McGarry \& Elkan, 2002) of residents in RACFs are experiencing regular pain. It is possible that these figures understate the occurrence of pain for RACF residents, because few studies have examined residents with dementia, who form a large proportion of RACF residents (Jorm, 2001). The consequences of persistent pain include impaired physical function, decreased mobility and psychological problems including depression and anxiety (Horgas \& Dunn, 2001; Won et al., 1999). Pain is generally multi-faceted in older adults who are likely to have co-morbidities that contribute to their pain experience (Ferrell, 1995; Keefe, Beaupre`, Weiner \& Seigler, 1996).The most common causes of pain include musculoskeletal disorders such as osteoarthritis, osteoporosis, bone factures and rheumatoid arthritis (Farrell, Gibson \& Helme, 1996; Gartell, 2005).

There are a number of barriers to the identification and effective management of pain for residents in RACFs (Higgins, Madjar \& Walton, 2004). A significant obstacle to effective pain management is the heavy reliance on nonprofessional staff for the provision of personal care (Herr, 2002; Horgas \& Dunn, 2001). In Australia, unregulated NAs have increasing responsibility for providing personal care to residents in RACFs (Richardson \& Martin, 2004). NAs provide care to residents under the supervision of a registered nurse (degree/diploma prepared nurses) or enrolled nurse (nurses with vocational education only), 
however, they often work alone and report to nursing staff infrequently (Rohr, Schneider \& Good, 2003). To date, there has been a paucity of research examining the NAs' experiences of caring for older residents in pain. The purpose of this study was to explore and describe the experiences of NAs who care for older residents in pain.

\section{Methods}

A qualitative approach applying a descriptive, exploratory method has been used to describe NAs' experience with caring for older residents in pain. A descriptive exploratory method is broad, purposive and designed to maximize discovery in the chosen area (Stebbins, 2001). This design facilitated thorough exploration and provided meaningful data that was easily interpreted. Ethical approval for the study was obtained from the Human Research Ethics Committee of Edith Cowan University and specific requirements of the participating organizations were also met.

\section{Sample}

A purposive, convenience sampling method was used to recruit NAs currently employed in RACFs in the South West of Western Australia. Participants were recruited through their workplaces. Advertisements and short information sessions were used to attract interest in the study. Interested individuals were given an information letter and were required to sign a declaration of informed consent. Participants were required to be NAs with at least six months experience, be able to speak English, and to have experience 
with the phenomenon under investigation (pain in older residents). The sampling process was guided by data collection, and continued until data saturation was achieved. Six NAs participated in this study.

\section{Procedure}

Data were collected through semi-structured, narrative style interviews. The interviews were between 20 and 40 minutes in duration and were audiotape recorded and transcribed verbatim and all identifying material was removed. Data collection commenced with a broad perspective and became increasingly focused as data analysis continued.

\section{Data Analysis}

A constant comparison method was employed and data collection and analysis occurred simultaneously to allow themes that captured the essence of the data to emerge (Patton, 1990). The data were analyzed using a three stage process. Initially data were sorted into codes that identified key concepts from words or phrases; 28 concepts were identified during coding. Codes were collapsed to form categories and finally categories were integrated to create two themes that reflected the experiences of the NAs. Verification of tentative findings occurred in follow up meetings with three participants.

The trustworthiness of qualitative research is demonstrated through the four checks of credibility, dependability, confirmability and transferability and these checks have been applied to this study (Polit \& Beck, 2006). The credibility of this study was enhanced by several techniques, including triangulation of data by 
using multiple participants from several care settings and using member checks by asking respondents to view initial findings. Dependability was demonstrated by coding being conducted by both authors independently and then compared, and by the second author confirming the emerging categories. A self-reflective journal was kept to document the researchers' assumptions and understandings and together with an audit trail that documented all theoretical decision making demonstrated confirmability of the study. Finally, three different sites were used to recruit participants and a variety of care experiences were sought using a purposive sampling method. This allowed rich description of the care setting to be provided so that transferability was demonstrated.

\section{Findings}

\section{Setting}

Three RACFs in regional Western Australia assisted with recruitment. Two of the facilities provided low level care (care provided to residents with who are largely independent but have some care needs) and the third provided high level care (care for residents who require extensive assistance with personal care and activities of daily living). Each facility provided care to more than 55 residents. Facilities differed in the type of staff employed: all three facilities employed registered nurses, but only one facility had registered nurses regularly available for clinical practice, with the other two using registered nurses for management and administrative purposes only. A greater number of enrolled nurses were employed in the low level care facilities and access to a registered nurse was on an on-call basis. 


\section{Participants}

Six NAs participated in this study. The demographic characteristics of the sample are presented in Table 1. Participants varied with regard to age, gender, educational background and duration of employment as a NA.

Table 1. Characteristics of the Sample

\begin{tabular}{|l|l|r|}
\hline $\begin{array}{l}\text { Characteristics } \\
\text { Sampled }\end{array}$ & $\begin{array}{l}\text { Variation within } \\
\text { Characteristics }\end{array}$ & $\begin{array}{l}\text { Number of participants } \\
(\mathbf{n}=6)\end{array}$ \\
\hline Age & $<25$ years & 0 \\
& $26-35$ years & 1 \\
& $>46-45$ years & 3 \\
& & 2 \\
\hline Gender & Female & 5 \\
& Male & 1 \\
\hline Education & Experience only & 2 \\
& Certificate III Aged Care & 1 \\
& Diploma of Aged Care & 1 \\
& Nursing Education (not & 2 \\
\hline Duration of & practicing nurses) & 1 \\
Employment as a & 0-5 years & 3 \\
Nursing Assistant in & 6-10 years & 1 \\
the Aged Care Sector & $>16$ years & 1 \\
\hline
\end{tabular}

\section{NAs' experiences}

The central theme that emerged from the data was Perfect Positioning. This theme describes the central role of NAs in RACFs and their how their unique situation allowed them to be involved in the pain management process. Five associated categories relate to the central theme: Frontline, Knowing the Resident, Emotional Attachment, Teamwork and Rewards of Getting It Right. In addition a sub-theme, Extended Roles, describes the extra responsibilities of 
NAs and includes two categories, Clinical Judgments and Nagging. The relationship between the themes is represented in Figure 1.

\section{Perfect Positioning}

Perfect Positioning describes NAs being ideally situated to assist in pain management process in RACFs. The facilities involved in this study were staffed primarily by NAs and consequently NAs worked most directly with residents and provided the majority of personal care. NAs were also uniquely situated to become a confidant of residents and were likely to be trusted with personal information. Regardless of the level of care provided by the facility, participants described working more closely with residents than other health care professionals. NAs acknowledged their central role in residents' care. One NA said:

Well certainly we are the first line. Because the RNs [registered nurses] and ENs [enrolled nurses] don't do the hands on so...so they're not there, and we are there when they [the resident] get out of bed, or when they transfer, so they [RNs and ENs] wouldn't know unless we told them. So it really is up to us to notice any changes (NA3). 


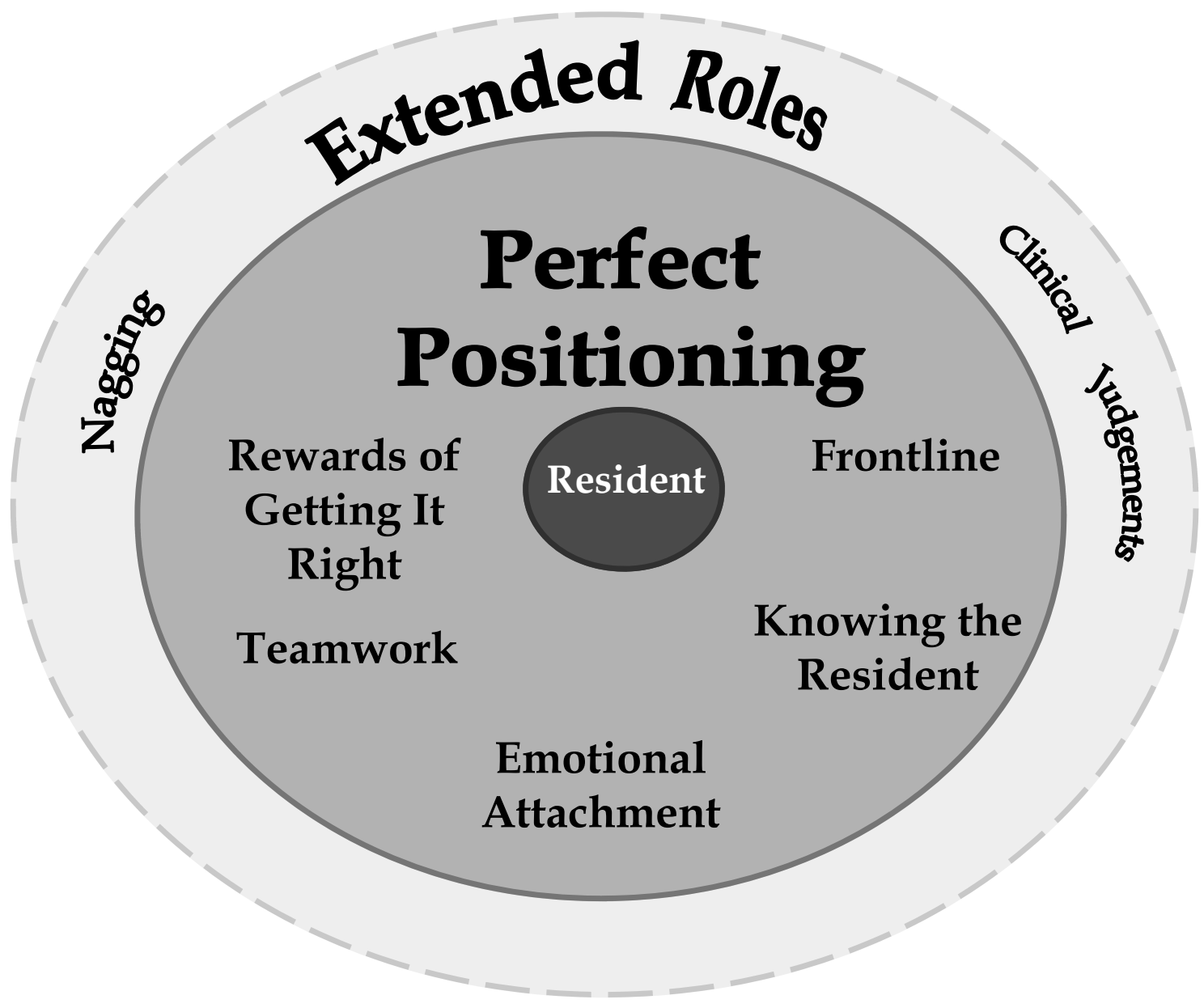

Figure 1. The nursing assistant's role in the management of pain.

\section{Frontline}

The category Frontline describes the positioning of NAs at the forefront of the pain management process in RACFs. The facilities involved in this study were staffed mainly by NAs, who provided all direct care for residents. Assisting residents to transfer, ambulate and provide support for other activities of daily living allowed NAs to notice changes in function and behavior that may be indicative of pain.

As well as NAs' primary role in the identification of a resident's pain, participants described having an important role in both the pharmacological and 
non-pharmacological management of pain. An NA spoke of her role in the pharmacological management of pain:

And generally if there is pain, we [NAs] can go into their [the residents] purse pack and there is PRN [as needed] medication. If it's ongoing pain management, then it generally goes into their regular pack [pharmacy packed medication] (NA3).

NAs also described initiating hot packs, massages, repositioning as non-pharmacological methods of pain management.

\section{Knowing the Resident}

Knowing the Resident describes how having knowledge of each resident is a key part of an NA's job and is an important factor in their ability to assess for pain and monitor effectiveness of interventions. NAs emphasized the importance of familiarizing themselves with residents' normal behaviors and habits. NAs reported that through knowing the resident, they improved their ability to notice changes and recognize behavior that may signify pain. Although NAs recognized the importance of care plans, which describe residents' capabilities, they emphasized the limitations of care plans for assisting staff to recognize pain in those individuals unable to express their discomfort. All participants told of their reliance on observing changes in behaviors that can be identified as relating to a resident's pain. One NA said:

...because a lot of them can't actually tell you, so you're really looking for these outward signs to do something (NA2).

The advantage of having continuity of staff was discussed by participants. Continuity of staff in facilities and particular areas was perceived as necessary to 
allow the opportunity to become familiar with residents, their families and other members of the health care team.

\section{Emotional Attachment}

Working closely with residents over extended periods allowed NAs to develop an attachment that may not have occurred in an acute or short-term care facility. The category Emotional Attachment describes the emotional bond formed between carers and residents.

RACFs are designed to provide care to older individuals in a home-like environment. NAs acknowledged the importance of privacy, respect and providing a home-like environment for residents, and discussed how working with residents on a daily basis they developed a relationship that could be equated to a family style bond. Several participants spoke of their parent or grandparent-like association with residents. The attachment was seen by NAs to be a two-way relationship. Not only did NAs become attached to residents, but they perceived that residents also became attached to them.

The emotional attachment between NAs and residents had both positive and negative consequences. Participants acknowledged that at times they felt emotionally exhausted by their role and found it distressing to see residents in pain. The family-like relationship allowed NAs to provide individualized care and for residents to feel at home. However, by not distinguishing between personal and professional boundaries, NAs described an emotional involvement that may have blurred the objectivity of their professional role. 


\section{Teamwork}

The category Teamwork describes the need to get support from the care team. The high dependency levels of residents meant that even simple activities of daily living required the assistance of more than one NA and NAs were therefore required to work closely with one another. All participants in this study emphasized the importance of the team environment. In particular, they reported that the team environment assisted them to solve problems together. NAs were often the only staff members working directly with residents and access to nursing staff was limited. Issues regarding residents were discussed among the team of NAs and decisions were made regarding whether to initiate interventions or to refer to an enrolled or registered nurse. One NA discussed how decisions were made regarding resident's care:

...pretty much it's a team thing....We all get on really well...Umm I think probably the [NAs] sort of draw off each other actually... Yeah we do really. If we are worried about things, we tend to talk to each other and bounce off each other (NA2).

There was a feeling expressed by some NAs that they were subservient to other staff and are not actively involved in the decisions made regarding residents' care. Some NAs perceived that they were largely excluded from the decision making process. One NA said:

Oh, I think as [NAs] we're at the bottom of the ladder, but we do the most important job. l'd like to see [NAs] become more involved in the pain management side of it...I mean we're the ones in the wards and spend the bulk of the time with the residents. That um, we might pick things up [but] after that we don't have anything more to do with what is organized. They [nurses] might just come in 
and say their [resident] medication has been changed and you might say, "Well ok this is what we're doing now" (NA4).

NAs conveyed their exclusion from decisions as being a reflection of the value of their role held by other health professionals. However, NAs maintained that because of their unique role working closely with residents they should be involved in clinical decisions relating to residents care.

\section{Rewards of Getting It Right}

The category Rewards of Getting It Right describes the way NAs are sustained and fulfilled by the rewarding aspects of caring for older individuals in pain. Each participant acknowledged the difficulties associated with caring for older people and particularly those unable to communicate their discomfort. However, all participants spoke of their passion, enjoyment and love for their work. Despite the emotional distress associated with observing people in pain, NAs gained satisfaction from seeing residents relieved of pain. One said:

Oh rewarding, yeah when they give you a smile or they are looking chirpy that's terrific and when they are happy that's great, 'cause that's what we're here for (NA2).

The satisfaction achieved from helping was the main reason that NAs remained in the role. NAs spoke of the poor financial remuneration they received and expressed the view that it was emotional fulfillment that made the job worthwhile. In recognizing the vulnerability of the resident population, the participants described having no expectation of being praised, instead identifying the fulfillment of seeing residents respond to the care that they provided. 


\section{Extended Roles}

The sub-theme Extended Roles describes the additional responsibilities of NAs in RACFs. Two categories emerged that are directly associated with the sub-theme, Clinical Judgments and Nagging. NAs described performing skilled tasks that were once the role of qualified staff, such as conducting assessments and administering medications. Participants in this study also described making clinical decisions, initiating pain management and pursuing other health professionals to order and carry out pain management interventions.

\section{Clinical Judgments}

Clinical Judgments describes the clinical decision making that was required of the NAs. Participants described being in a position where they were required to make judgments about a resident's pain management, and in particular were expected to identify residents who were in pain. Without formal training in pain assessment, NAs relied on their previous knowledge of residents to enable them to assess a resident's current pain status.

Once pain was identified by the NA, it was their responsibility to decide on the course of action to be taken. NAs described three options that were available to them. These were to provide NA driven interventions, communicate the problem to nurses, or to ignore the problem (see Figure 2). NAs had to make the ultimate decision about which option to take, with little or no guidance from others. 


\section{Nagging}

Nagging describes the continual pursuit of pain management for residents, often requiring them to hound other staff for action. Nagging also encompasses a concept of ownership of pain management issues by NAs. Several participants perceived that pain management was often not a high priority for nurses and NAs therefore felt that they had to 'nag' nurses to get appropriate interventions for residents. One participant said:

Yeah, its mainly nagging at nurses who then have to nag at doctors to come (laugh) it's a never ending circle. Nag, nag, nag, and eventually everyone gets sick of you and they eventually cave in and come and do something (NA2).

NAs described advocating for residents as an important part of their role in pain management in RACFs. NAs took ownership of the pain management problem and felt it was their responsibility to follow it up.

\section{Discussion}

\section{The role of NAs}

The pivotal role of NAs working with residents has been described previously (Ferrell, 1996; Herr, 2002; Horgas \& Dunn, 2001). The amount of time that NAs spend with residents allows greater opportunity to identify residents in pain than may be possible for other groups of aged care workers. This study has described the central role in pain management that NAs in RACFs have. However, it should be noted that it was not the expectation of the participating RACFs that NAs manage pain or that nurses are delegating care related to pain 
management to NAs. Instead, NAs were often the only staff available to perform such a role.

Identification of Resident's Pain

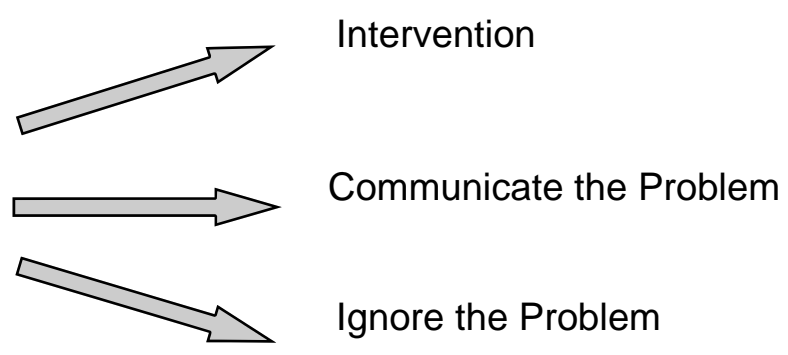

Figure 2. Clinical decision-making process for nursing assistants.

In Western Australia a NAs' role is to provide general, basic care under the direct supervision and delegation of registered and enrolled nurses (Nurses Board of Western Australia [NBWA], 2006). However, NAs represent a majority in the RACF workforce and in some RACFs nurses are not on the premises at all times. Therefore, it is essential to more clearly define the role of NAs working in RACFs, to ensure that professional boundaries are defined for the provision of safe and legally appropriate care for RACF residents.

\section{Educational Preparation}

NAs in Australia, America and the United Kingdom have minimal education and training to support them in an expanding role in RACFs (Allcock et al., 2002; Davies et al., 1999; Neville et al., 2006). Two thirds of participants in this study had commenced employment as a NA without any relevant formal qualification, education or training, although all had since completed, or were currently completing, relevant education in aged care. This study supports the literature that suggests that lack of education and understanding by NAs may impact on 
the effectiveness of pain management in RACFs, because NAs may not have the necessary knowledge or skills to accurately assess pain and implement appropriate interventions (Allcock et al., 2002; Ferrell, 1996; Twycross, 2002).

Previous studies, although having a nursing focus, have concluded that education about pain increases staff knowledge levels and can have a positive influence on staff behaviors (Allcock et al., 2002; Twycross, 2002). However, increasing NAs' pain management knowledge may not be sufficient to improve the management of pain in RACFs and the degree to which education may influence the practice of NAs is largely unknown (Briggs \& Dean, 1998). Hence, the findings of this study support suggestions by Horgas and Dunn (2001) that increased access to training and the implementation of mandatory education related to pain assessment and management for NAs employed in RACFs may be valuable. It is noteworthy that care assistants have to 'nag' at nurses and medical practitioners to get appropriate treatments for residents, despite pain management not being within their scope of practice. Several studies have shown that nurses and medical practitioners are deficient in general knowledge about providing pain management to older people and are in need of pain education (Herr, 2002; Herr \& Mobily, 1996; Tarzian \& Hoffmann, 2004). It is possible that trained staff are neglecting this vital area of resident care, and further research is required to determine the attitudes of nurses and medical practitioners to providing care to older residents in pain. 
It is possible that pain may not considered a high priority by health professionals, but is considered more important by staff who work closely with residents and are more aware of the impact of unrelieved pain. Although NAs do not have adequate training in pain assessment and management, it is possible that they are the most vocal advocates for residents in pain. Further research is needed to highlight the care priorities of health professionals working in aged care and compare them with resident concerns.

\section{Continuity of Staff and Care}

The intimate nature of tasks performed by NAs in long-term care facilities enables NAs to develop complex knowledge of residents. In several studies NAs have described knowing the residents and their usual behaviors as important factors in determining a resident's pain (Mentes, Teer \& Codogan, 2004; Wright, Varholak \& Costello, 2003). Pain assessment tools such as the Abbey Pain Scale consider that a health providers' knowledge of a resident is a crucial factor in the timely identification of pain (Abbey et al., 2004).

Continuity of staff was seen by NAs in this study to be a determining factor in the development of familiarity and knowledge of residents. The importance of continuity of staff for consistent, high quality care in RACFs has been identified in previous studies and is also inherent in the Australian Government Aged Care Act of 1997 (Allcock et al., 2002; Commonwealth of Australia, 2005). It can be difficult to maintain staff in the aged care sector because of poor pay and conditions offered to workers (Chou, Boldy \& Lee, 2002). RACFs often rely on agency or casual staff due to unpredictable fluctuations in labor needs 
(Richardson \& Martin, 2004). The reliance on non-permanent staff members diminishes the ability of workers to know residents and may impact on the quality of care provided to residents. The findings of this study support the implementation of incentives to recruit and retain staff in the aged care sector in an effort to improve resident care, including pain management.

\section{The Future of Nursing Assistants in Residential Aged Care Facilities}

The change in delivery of services in the Australian health care system in recent years has meant an increased reliance on unlicensed health care workers in the aged care sector (NBWA, 2006). The greater demand for aged care services has resulted in an increase in NAs' roles and responsibilities in the residential aged care setting (Ferrell, 1996; Richardson \& Martin, 2004). This study reports the increase in duties delegated to NAs in RACFs in relation to pain management and the requirement for clinical judgments to be made by NAs. It is imperative that the role of NAs in RACFs is examined to determine the practical and legal implications of current practice.

Despite the expansion in the level of clinical practice of NAs in RACFs, there has been minimal change in the level of education required to prepare NAs for their work role. Perhaps more problematic is the lack of a defined scope of practice and regulatory body for NAs in Australia. The need for a defined scope of practice for NAs is evident, because NAs perform tasks and make clinical judgments about residents' care that would traditionally have been considered a nursing duty. Scope of practice documents relating to NAs would provide 
boundaries for practice, would govern appropriate delegation from health

professionals to NAs and guide the provision of safe care (NBWA, 2006).

\section{Limitations of the Study}

The time and financial constraints of the study limited the sample and size of the study, which consequently influenced the data collected. However, a purposive approach to sampling obtained rich and in-depth data from NAs and data saturation was achieved. The study was restricted to the South West of Western Australia, however this study has provided a regional perspective that has been neglected in previous studies. Despite these limitations this study has contributed to health knowledge and has implications for future research, education and practice.

\section{Conclusion}

This study has illustrated the complex and pivotal role NAs have in the assessment and management of pain for residents in RACFs. NAs represent a majority of the RACF workforce and it is apparent that they are often responsible for initiating and implementing pain management interventions to address the needs of older residents in their care. The complexity of tasks performed by some NAs underscores the need to adequately prepare them through education and training, to ensure that they have the necessary knowledge and skills to perform this role. Furthermore the escalation in the level of clinical practice performed by NAs' depicted in this study, emphasize the need for a defined scope of practice and national regulatory body for NAs in Australia. 


\section{Acknowledgements}

This study was conducted in partial fulfillment of the requirements of an honors degree. The authors acknowledge the financial support of the Royal College of Nursing, Australia. The authors also acknowledge with thanks the assistance of the participating residential aged care facilities and the NAs who volunteered their time for this study. 


\section{REFERENCES}

Abbey, J., Piller, N., DeBellis, A., Esterman, A., Parker, D., Giles, L., et al. (2004). The abbey pain scale: A 1-minute numerical indicator for people with end stage dementia. International Journal of Palliative Nursing, 10(1), 6-13.

Allcock, N., McGarry, J., \& Elkan, R. (2002). Management of pain in older people within the nursing home: A preliminary study. Health and Social Care in the Community, 10(6), 464-471.

The Australian Pain Society [APS]. (2005). Pain in Residential Care Facilities: Management Strategies. North Sydney, NSW: Author.

Briggs, M., \& Dean, K.L. (1998). A qualitative analysis of the nursing documentation of postoperative pain management. Journal of Clinical Nursing, 7, 155-163.

Chou, S., Boldy, D., \& Lee, A. (2002). Staff satisfaction and its components in residential aged care. International Journal for Quality in Health Care, 14, 207-217.

Commonwealth of Australia. (2005). Australian Aged Care Act. 1997, No.112 of 1997. Retrieved $6^{\text {th }}$ December, 2006, from 
http://www.health.gov.au/internet/wcms/publishong.nsf.content/ageing$\underline{\text { legislat-aca1997 }}$

Davies, S., Slack, R., Laker, S., \& Philip, I. (1999). The educational preparation of staff in nursing homes: Relationship with resident autonomy. Journal of Advanced Nursing, 29(1), 208-217.

Farrell, M. J, Gibson, S.J., \& Helme, R.D. (1996). Chronic non-malignant pain in older people. In B. Ferrell \& B. Ferrell (Eds.), Pain in the elderly: Task force on pain in the elderly. Seattle: International Association for the Study of Pain [IASP] Press.

Ferrell, B. A. (1996). Overview of aging and pain. In B. Ferrell \& B. Ferrell (Eds.), Pain in the elderly: Task force on pain in the elderly. Seattle: International Association for the Study of Pain [IASP] Press.

Ferrell, B. A. (1995). Pain evaluation and management in the nursing home: Diagnosis and treatment. Annals of Internal Medicine, 123(9), 681-687.

Gartell, M. (2005). Expression of description of chronic pain by older people. Australasian Journal on Ageing, 24(1), 33-37.

Herr, K. (2002). Chronic pain: Challenges and assessment strategies. Journal of Gerontological Nursing, 28(1), 20-30. 
Herr, K., \& Mobily, P. (1996). Pain management for the elderly in the alternate care setting. In B. Ferrell \& B. Ferrell (Eds.), Pain in the elderly: Task force on pain in the elderly. Seattle: International Association for the Study of Pain [IASP] Press.

Higgins, I., Madjar, I., \& Walton, J. (2004). Chronic pain in elderly nursing home residents: The need for nursing leadership. Journal of Nursing Management, 12(3), 167-173.

Horgas, A., \& Dunn, K. (2001). Pain in nursing home residents: Comparison of residents' self report and NAs' perceptions. Journal of Gerontological Nursing, 27(3), 44-56.

Jorm, A. (2001). Dementia: A major health problem for Australia. Perth: Alzheimer's Association of Australia.

Keefe, F., Beaupre`, P., Weiner, D. \& Seigler, I. (1996). Pain in older adults: A cognitive behavioral perspective. In B. Ferrell \& B. Ferrell (Eds.), Pain in the elderly: Task force on pain in the elderly. Seattle: International Association for the Study of Pain [IASP] Press. 
Mentes, J.C., Teer, J., \& Codogan, M.P. (2004). The pain experience of cognitively impaired nursing home residents: Perceptions of family members and certified NAs. Pain Management Nursing, 5(3), 118-125.

Neville, C., McCarthy, A., \& Laurent, K. (2006). Pain management skills of regional nurses caring for older people with dementia: A needs analysis. Collegian, 13(2), 31-36.

Nurses Board of Western Australia [NBWA]. (2006). Position statement on unlicensed health care worker in Western Australia. Retrieved $5^{\text {th }}$ December, 2006, from http://nbwa.org.au

Patton, M. Q. (1990). Qualitative evaluation and research methods ( $2^{\text {nd }}$ ed.). Newbury Park: Sage Publications.

Polit, D., \& Beck, C. (2006). Essentials of nursing research: Methods, appraisal and utilization ( $6^{\text {th }}$ ed.). Philadelphia: Lippincott.

Richardson, S., \& Martin, B. (2004). The care of older Australians: A picture of the residential aged care workforce. Adelaide: National Institute of Labour Studies, Flinders University. 
Rohr, Y., Schneider, J., Good, P., \& Sattler, L. (2003). Availability of analgesia for breakthrough pain for palliative care residents in hostels. Geriaction, 21(4), 11-16.

Stebbins, R.A. (2001). Exploratory research in the social sciences. Thousand Oaks, C.A.: A Sage University Paper.

Twycross, A. (2002). Educating nurses about pain management: The way forward. Journal of Clinical Nursing, 11, 705-714.

Tarzian, A.J., \& Hoffmann, D.E. (2004). Barriers to managing pain in the nursing home: Findings from a statewide survey. Journal of the American Medical Directors Association, 5(2), 82-8.

Won, A., Lapane, K., Gambassi, G., Bernabei, R., Mor, V., \& Lipsitz, L. (1999). Correlates and management of non-malignant pain in the nursing home. American Geriatrics Society, 47(8), 936-942.

Wright, J., Varholak, D., \& Costello, J. (2003). Voices from the margin: The nurse aide's role in pain management of institutionalized elders. American Journal of Alzheimer's disease and other Dementia, 18(3), 154-158. 